University of Nebraska - Lincoln DigitalCommons@University of Nebraska - Lincoln

\title{
Poroacoustic waves under a mixture-theoretic based reformulation of the Jordan-Darcy-Cattaneo model
}

P. M. Johnson

U.S. Naval Research Laboratory, pedro.jordan@nrlssc.navy.mil

F. Passarella

Università di Salerno

V.Tibullo

Università di Salerno

Follow this and additional works at: http:// digitalcommons.unl.edu/usnavyresearch

Johnson, P. M.; Passarella, F.; and Tibullo, V., "Poroacoustic waves under a mixture-theoretic based reformulation of the Jordan-Darcy-Cattaneo model" (2017). U.S. Navy Research. 130.

http://digitalcommons.unl.edu/usnavyresearch/130

This Article is brought to you for free and open access by the U.S. Department of Defense at DigitalCommons@University of Nebraska - Lincoln. It has been accepted for inclusion in U.S. Navy Research by an authorized administrator of DigitalCommons@University of Nebraska - Lincoln. 


\title{
Poroacoustic waves under a mixture-theoretic based reformulation of the Jordan-Darcy-Cattaneo model
}

\author{
P.M. Jordan ${ }^{\mathrm{a}, *}$, F. Passarella ${ }^{\mathrm{b}}$, V. Tibullo ${ }^{\mathrm{b}}$ \\ a Acoustics Division, U.S. Naval Research Laboratory, Stennis Space Center, MS 39529, USA \\ b Dipartimento di Matematica, Università di Salerno, Fisciano, Italy
}

\section{H I G H L I G H T S}

- Present a reformulation of the Jordan-Darcy-Cattaneo (JDC) poroacoustic model.

- Derive acceleration wave and traveling wave results from reformulated JDC model.

- Resolve issues raised by the acceleration wave analysis of Ciarletta et al. (2013).

\section{A R T I C L E I N F O}

\section{Article history:}

Received 10 April 2016

Received in revised form 30 July 2016

Accepted 31 July 2016

Available online 10 August 2016

\section{Keywords:}

Jordan-Darcy-Cattaneo model

Poroacoustic phenomena

Acceleration waves

Traveling waves

Mixture theory

\begin{abstract}
A B S T R A C T
Using mixture theory, we present a reformulation of the Jordan-Darcy-Cattaneo (JDC) poroacoustic model. The one-dimensional (1D) version of the resulting system is then applied to the description of first 'start-up' acceleration waves, and then those exhibited under the traveling wave reduction, in a gas that saturates a rigid, porous solid. Results obtained are contrasted with those of the Darcy-Jordan model, the impact of $\tau$, the model's relaxation time parameter, is assessed, and issues stemming from the acceleration wave analysis performed by Ciarletta et al. (2013), who proposed the original JDC model, are resolved. Also, the weakly-nonlinear Darcy-Jordan model is generalized to include the case $\tau>0$.
\end{abstract}

Published by Elsevier B.V.

\section{Introduction}

In 2013, Ciarletta et al. [1] investigated poroacoustic acceleration waves in fluids that saturate a class of porous solids that are both rigid and admit porosity values in the range $0.1 \lesssim \chi \lesssim 0.4$, where we note that, in general, $\chi \in(0,1)$. In such media, the authors of Ref. [1] contend, poroacoustic propagation is more accurately modeled not by the conventional 'Darcy-Jordan model' (DJM) [2,3], but instead by the following first order system, which they term the 'Jordan-Darcy-Cattaneo' (JDC) model:

$$
\begin{aligned}
& \frac{D \varrho}{D t}+\varrho(\nabla \cdot v)=0, \\
& \frac{\mu \chi}{K}\left(\tau \frac{\mathfrak{D} \boldsymbol{v}}{\mathfrak{D} t}+\boldsymbol{v}\right)=-\nabla P,
\end{aligned}
$$

\footnotetext{
* Corresponding author.

E-mail address: pedro.jordan@nrlssc.navy.mil (P.M. Jordan).
} 
which they augment with a barotropic, but otherwise arbitrary, equation of state. In Sys. (1), $v=\mathbf{v}-\mathbf{v}_{\mathrm{s}} / \chi$, where $\mathbf{v}=(u, v, w)$ is the intrinsic average velocity of the fluid [4, Section 1.3] and $\mathbf{v}_{s}$ is the velocity ${ }^{1}$ of the solid matrix; $\varrho(>0)$ is the mass density of the fluid; $P$ is a pressure; $\mu(>0)$ is the shear viscosity; $K(>0)$ is the permeability; $\tau(>0)$, the relaxation time parameter, distinguishes Eq. (1b) from Darcy's law in that letting $\tau \rightarrow 0$ reduces the former to the latter; $D / D t$ denotes the material derivative operator; and $\mathfrak{D} / \mathfrak{D} t$, the Lie derivative operator of 'Christov-Morro' theory [6-8], becomes in the present setting (see Ref. [1, Eq. (5)])

$$
\frac{\mathfrak{D} v}{\mathfrak{D} t}:=\frac{\partial \boldsymbol{v}}{\partial t}+(\nabla \cdot v) \boldsymbol{v}
$$

Throughout their analysis, Ciarletta et al. [1] tacitly regard Eq. (1b) not as a constitutive relation for the drag force but, rather, as the momentum equation for the fluid phase; and indeed, its form echoes that of the DJM, as can be seen by reexpressing Eq. (1b) as

$$
\frac{\mathfrak{D} v}{\mathfrak{D} t}=-\left(\frac{1}{\varrho^{*}}\right) \nabla P-\left(\frac{1}{\tau}\right) \boldsymbol{v},
$$

where we have set $\varrho^{*}:=\tau \mu \chi / K$ for convenience, and we note that all material parameters are taken as constants in Ref. [1]. (If we replace $\mathfrak{D} / \mathfrak{D} t$ and $\varrho^{*}$ with $D / D t$ and $\varrho$, respectively, Eq. (3) becomes the Euler momentum equation subjected to the external, velocity-dependent (per unit mass) body force ${ }^{2}-\boldsymbol{v} / \tau$ ). Sys. (1) is, therefore, inconsistent with the mixture theory-based approach of Rajagopal and Tao [9], who point out that porous media drag (i.e., filtration) laws such as Darcy's law are 'mere approximations' to the balance of linear momentum for the fluid phase. The analysis of Ciarletta et al. is further impacted by the fact that, under their formulation, $P$ is treated as the thermodynamic pressure (i.e., as being given by an equation of state), not the intrinsic quantity it represents in the context of filtration laws; see Ref. [4, Section 1.4.1].

In this article, we consider poroacoustic wave phenomena under a model system posited on Eq. (1b). However, while we assume the same filtration law as Ciarletta et al., the model presented below differs from that put forth by the authors of Ref. [1] because it is formulated under the mixture-theoretic approach described in Ref. [9]. The primary aim of our investigation is to generalize the acceleration wave results of Jordan [3] and Jordan and Fulford [10], who assumed Darcy's law, to include the effects of $\tau$. Our analysis, however, also resolves a suspect finding noted by Ciarletta et al. (see Ref. [1, Section 4]) regarding acceleration wave amplitudes under the 1D version of Sys. (1) and another regarding the wave speed expression given in Ref. [1, Eq. (15)]

To this end, the exposition presented below is organized as follows. In Section 2, the balance laws and constitutive assumptions of our reformulation of the JDC model are stated and the resulting system of equations is then specialized to the case of irrotational flow. In Section 3, two versions of the weakly-nonlinear equation of motion (EoM) corresponding to the reformulated JDC system are derived and briefly discussed. In Section 4, an analysis of 'start-up' acceleration waves is carried out (in 1D) and the above-noted difficulties with the findings of Ciarletta et al. are resolved. Then, in Section 5, a limited traveling wave analysis of the reformulated DJC model is performed, with a focus on acceleration waves, and we contrast our results with those derived in Ref. [10] under the exact DJM. Lastly, in Section 6, we summarize our findings and note possible extensions of the present model.

Remark 1. It is noteworthy that the Oldroyd-inspired relation

$$
\left(1+\lambda_{v} \frac{\partial}{\partial t}\right) \mathbf{v}=-\frac{K}{\mu \chi}\left(1+\lambda_{P} \frac{\partial}{\partial t}\right) \nabla P,
$$

where $\lambda_{v}$ and $\lambda_{P}$ are, respectively, relaxation and retardation time constants, has been suggested as a filtration law for viscoelastic fluid flow in porous media; see Ref. [11, Section 1] and those cited therein.

\section{Balance laws and constitutive relations}

Consider the following system describing, under the homentropic [12] assumption, the flow of a perfect gas ${ }^{3}$ that permeates a rigid, homogeneous and isotropic, porous solid, the drag of which on the gas being described by Eq. (1b):

$$
\frac{D \varrho}{D t}+\varrho(\nabla \cdot \mathbf{v})=0
$$

\footnotetext{
1 In Ref. [1], the $1 / \chi$ coefficient of $\mathbf{v}_{\mathrm{s}}$ appears to have been inadvertently omitted; this, however, is not an issue since, as noted in Ref. [5, Section 3.1], the assumption that the solid phase is rigid means that $\mathbf{v}_{\mathrm{s}}=\mathbf{0}$ can be taken without error nor loss of generality.

2 To avoid confusion in later sections, we hereafter adopt the terminology of Rajagopal and Tao [9] when discussing Sys. (5) below.

3 As defined by Thompson [12, Section 2.5], an ideal gas in which $c_{\mathrm{p}}>c_{\mathrm{v}}>0$, the specific heats at constant pressure and volume, respectively, are constants; Whitham [13, Section 6.4], however, notes that such ideal gases are sometimes referred to as polytropic gases.
} 


$$
\begin{aligned}
& \varrho \frac{D \mathbf{v}}{D t}=-\nabla \wp-\frac{\mu \chi}{K}\left[\tau\left(\frac{D \mathbf{v}}{D t}-(\mathbf{v} \cdot \nabla) \mathbf{v}+(\nabla \cdot \mathbf{v}) \mathbf{v}\right)+\mathbf{v}\right], \\
& \wp=\wp_{0}\left(\varrho / \varrho_{0}\right)^{\gamma},
\end{aligned}
$$

where here and henceforth $\mathbf{v}_{\mathrm{s}}=\mathbf{0}(\Rightarrow \boldsymbol{v}=\mathbf{v})$ is assumed; recall Footnote 1. In Sys. (5), $\gamma=c_{\mathrm{p}} / c_{\mathrm{v}}$, where $\gamma \in(1,5 / 3]$ is known as the adiabatic exponent; $\mu, \tau, K$, and $\chi$ are assumed to be constants, as in Ref. [1]; and the positive constants $\wp_{0}$ and $\varrho_{0}$ denote the equilibrium state values of $\wp(>0)$, the thermodynamic pressure, and $\varrho$, respectively.

With regard to reconciling the terms in Rajagopal and Tao's [9] formulation with those of the present study, we note the following: $\mathbf{m}$, the interaction term, is set equal to the left-hand side (LHS) of Eq. (1b); the inertia term, $\rho^{f} d \mathbf{v}^{f} / d t$, corresponds to the LHS of Eq. (5b); the partial stress is $\sigma^{f}=-\wp \mathbf{I}$, where I denotes the $3 \times 3$ identity tensor in Ref. [9]; and $\mathbf{b}=\mathbf{0}$, i.e., all external body forces are neglected. We also observe that Eq. (5b) contains only one type of temporal differential operator, namely, the material derivative, a simplification easily achieved by expressing $\mathfrak{D} \mathbf{v} / \mathfrak{D} t$ in terms of $D \mathbf{v} / D t$.

On rearranging terms and making use of the well known identity

$$
(\mathbf{v} \cdot \nabla) \mathbf{v}=\frac{1}{2} \nabla|\mathbf{v}|^{2}-\mathbf{v} \times(\nabla \times \mathbf{v}),
$$

Eq. (5b) becomes, after eliminating $\wp$ using Eq. (5c),

$$
\begin{aligned}
& \left(\frac{\tau \nu \chi}{K}+\varrho / \varrho_{0}\right)\left[\frac{\partial \mathbf{v}}{\partial t}+\frac{1}{2} \nabla|\mathbf{v}|^{2}-\mathbf{v} \times(\nabla \times \mathbf{v})\right]=-\gamma^{-1} c_{0}^{2} \nabla\left[\left(\varrho / \varrho_{0}\right)^{\gamma}\right] \\
& -\left(\frac{\nu \chi}{K}\right) \mathbf{v}+\frac{\tau \nu \chi}{K}\left[\frac{1}{2} \nabla|\mathbf{v}|^{2}-\mathbf{v} \times(\nabla \times \mathbf{v})-(\nabla \cdot \mathbf{v}) \mathbf{v}\right] .
\end{aligned}
$$

Here, we have introduced $c_{0}=\sqrt{\gamma \wp_{0} / \varrho_{0}}$, the adiabatic sound speed, which represents the speed of sound in the undisturbed gas, and $v=\mu / \varrho_{0}$ is the kinematic viscosity of the gas.

Henceforth restricting our attention to acoustic phenomena (i.e., assuming the flow to be irrotational), Eq. (7) is reduced to

$$
\begin{aligned}
\left(\frac{\tau \nu \chi}{K}+\varrho / \varrho_{0}\right) \nabla\left(\frac{\partial \phi}{\partial t}+\frac{1}{2}|\nabla \phi|^{2}\right)= & -\gamma^{-1} c_{0}^{2} \nabla\left[\left(\varrho / \varrho_{0}\right)^{\gamma}\right]-\left(\frac{\nu \chi}{K}\right) \nabla \phi \\
& +\frac{\tau \nu \chi}{K}\left[\frac{1}{2} \nabla|\nabla \phi|^{2}-(\nabla \phi) \nabla^{2} \phi\right] \quad(\nabla \times \mathbf{v}=\mathbf{0}) .
\end{aligned}
$$

Here, we have used the fact that $\nabla \times \mathbf{v}=\mathbf{0}$ implies $\mathbf{v}=\nabla \phi$, where $\phi$ denotes the scalar velocity potential.

Now introducing the following non-dimensional variables:

$$
\mathbf{v}^{\circ}=\frac{\mathbf{v}}{V_{\mathrm{c}}}, \quad \phi^{\circ}=\frac{\phi}{V_{\mathrm{c}} L}, \quad \nabla^{\circ}:=L(\nabla), \quad t^{\circ}=\left(\frac{c_{0}}{L}\right) t, \quad s=\frac{\varrho-\varrho_{0}}{\varrho_{0}},
$$

where $s$ is known as the condensation and $V_{\mathrm{c}}(>0)$ and $L(>0)$ respectively represent a characteristic speed and length in the particular problem under consideration, Eqs. (8) and (5a) become

$$
\begin{aligned}
\frac{\partial s}{\partial t}+\epsilon \nabla \cdot[(1+s) \nabla \phi]=0, & \\
\sigma(1+s / \sigma) \nabla\left(\frac{\partial \phi}{\partial t}+\frac{1}{2} \epsilon|\nabla \phi|^{2}\right)= & -(\epsilon \gamma)^{-1} \nabla\left[(1+s)^{\gamma}\right]-\delta \nabla \phi \\
& +\epsilon \operatorname{Sg}\left[\frac{1}{2} \nabla|\nabla \phi|^{2}-(\nabla \phi) \nabla^{2} \phi\right] \quad(\nabla \times \mathbf{v}=\mathbf{0})
\end{aligned}
$$

respectively. Here, $\epsilon:=V_{\mathrm{c}} / c_{0}$ is the Mach number; $\mathrm{Sg}:=\varrho^{*} / \varrho_{0}=\tau \nu \chi / K$ plays the role of what some authors refer to as the Straughan number, where we hereafter assume $\mathrm{Sg} \ll 1$ based on the perturbative nature of Eq. (1b); we have set $\sigma:=1+\mathrm{Sg}$; as in Ref. [3], the dimensionless Darcy coefficient is given by

$$
\delta=\frac{v \chi L}{K c_{0}}
$$

and all (o) superscript have been suppressed but should remain understood. 


\section{Derivation of weakly-nonlinear, bi-directional, model equations}

Invoking in this section the finite-amplitude approximation, ${ }^{4}$ the fundamental assumptions of which are $\epsilon \ll 1$ and $|s|=\mathcal{O}(\epsilon)$, we can, with the aid of the binomial theorem, rewrite Eq. (11) as

$$
\begin{aligned}
\nabla\left(\frac{\partial \phi}{\partial t}+\frac{1}{2} \epsilon|\nabla \phi|^{2}\right)= & -\hat{\delta}\left(1-s / \sigma+s^{2} / \sigma^{2}+\cdots\right) \nabla \phi \\
& -(\epsilon \sigma)^{-1}\left(1-s / \sigma+s^{2} / \sigma^{2}+\cdots\right) \nabla\left[s+\frac{1}{2}(\gamma-1) s^{2}+\cdots\right] \\
& +\epsilon \sigma^{-1} \operatorname{Sg}\left(1-s / \sigma+s^{2} / \sigma^{2}+\cdots\right)\left[\frac{1}{2} \nabla|\nabla \phi|^{2}-(\nabla \phi) \nabla^{2} \phi\right] .
\end{aligned}
$$

Here, we have set $\hat{\delta}:=\delta / \sigma$, and we observe that $\tau>0$ implies $\sigma>1$.

If it is also true that $\hat{\delta}=\mathcal{O}(\epsilon)$ and $\mathrm{Sg}=\mathcal{O}(\epsilon)$, then on simplifying and neglecting all terms of $\mathcal{O}\left(\epsilon^{2}\right)$ Eq. (13) is reduced to

$$
\nabla\left\{\left(\frac{\partial \phi}{\partial t}+\frac{1}{2} \epsilon|\nabla \phi|^{2}\right)+(\epsilon \sigma)^{-1}\left[s+\frac{1}{2}\left(\gamma-1-\sigma^{-1}\right) s^{2}\right]+\hat{\delta} \phi\right\}=0,
$$

which we can immediately recast as, after applying $\partial / \partial t$ to both sides,

$$
\phi_{t t}+\frac{1}{2} \epsilon \partial_{t}|\nabla \phi|^{2}+(\epsilon \sigma)^{-1}\left[1+\left(\gamma-1-\sigma^{-1}\right) s\right] s_{t}+\hat{\delta} \phi_{t}=0,
$$

where, in addition to a subscript ' $t$ ', we now use $\partial_{t}$ to denote $\partial / \partial t$. In obtaining Eq. (15), the resulting function of integration was set to zero without loss of generality.

Now using Eq. (10) to eliminate $s_{t}$, Eq. (15) becomes

$$
\phi_{t t}+\frac{1}{2} \epsilon \partial_{t}|\nabla \phi|^{2}+\hat{\delta} \phi_{t}=\sigma^{-1}\left[1+\left(\gamma-1-\sigma^{-1}\right) s\right]\left[(1+s) \nabla^{2} \phi+(\nabla s) \cdot(\nabla \phi)\right] .
$$

Finally, on eliminating $s$ using the well known relation (see, e.g., Ref. [14, Section 2])

$$
s=-\epsilon \phi_{t}+\mathcal{O}\left(\epsilon^{2}\right),
$$

and then neglecting all resulting terms of $\mathcal{O}\left(\epsilon^{2}\right)$, Eq. (16) becomes, after simplifying and rearranging terms, what might be termed the 'Blackstock' version of our EoM, specifically,

$$
\phi_{t t}-\hat{\mathfrak{a}}_{0}^{2}\left[1-\epsilon(\gamma-1 / \sigma) \phi_{t}\right] \nabla^{2} \phi+\hat{\delta} \phi_{t}+\frac{1}{2} \epsilon(1+1 / \sigma) \partial_{t}|\nabla \phi|^{2}=0
$$

where $\hat{\mathfrak{a}}_{0}=1 / \sqrt{\sigma}$ denotes the (dimensionless) sound speed in the undisturbed saturating gas. Here, we observe that $\hat{\mathfrak{a}}_{0}$ is an effective quantity in the sense that its value is affected by the presence of the porous matrix; specifically, the reformulated JDC model predicts a value of this parameter that is less, by a factor of $1 / \sqrt{\sigma}$, than that of the DJM [3]. In terms of dimensional quantities we have $\hat{c}_{0}<c_{0}$, where in the case of perfect gases

$$
\hat{c}_{0}=\frac{c_{0}}{\sqrt{\sigma}}=\sqrt{\frac{\gamma \wp_{0}}{\sigma \varrho_{0}}} .
$$

Here, we observe that $\hat{\mathfrak{a}}_{0}=\hat{c}_{0} / c_{0}$ and that $\hat{c}_{0} \rightarrow c_{0}$ as $\tau \rightarrow 0$. Additionally, one can define an 'effective $\gamma$ ' as $\gamma_{\text {eff }}=\gamma / \sigma$, where of course $\gamma_{\text {eff }}<\gamma$, meaning that $\gamma_{\text {eff }}<1$ may be possible, especially in the case of gases exhibiting a large number of degrees of freedom [12, p. 80].

If, on the other hand, we divide Eq. (18) by $\left[1-\epsilon(\gamma-1 / \sigma) \phi_{t}\right]$ and then expand in powers of $\epsilon$, where we recall the assumption $\epsilon \ll 1$, we get, after neglecting terms of $\mathcal{O}\left(\epsilon^{2}\right)$ and simplifying, the 'Kuznetsov' version of our EoM, namely,

$$
\phi_{t t}-\hat{\mathfrak{a}}_{0}^{2} \nabla^{2} \phi+\hat{\delta} \phi_{t}=-\frac{1}{2} \epsilon \partial_{t}\left[(1+1 / \sigma)|\nabla \phi|^{2}+(\gamma-1 / \sigma)\left(\phi_{t}\right)^{2}\right] .
$$

Here, we observe that Eq. (20) reduces to Jordan's [3, Eq. (2.16)], which has come to be known as the (weakly-nonlinear) DJM, on letting $\sigma \rightarrow 1$ (i.e., letting $\tau \rightarrow 0$ ); the parameter $\beta$, known as the coefficient of nonlinearity [15], appearing in the latter is related to $\gamma$ via $\beta=(\gamma+1) / 2$ when the fluid in question is a perfect gas.

We leave it to the reader to show that using the approximation $|\nabla \phi|^{2} \approx \hat{\mathfrak{a}}_{0}^{-2}\left(\phi_{t}\right)^{2}[16]$ on the right-hand side (RHS) of Eq. (20) yields the 'Lighthill-Westervelt' version of our EoM.

Remark 2. As they were derived under the finite-amplitude approximation, the PDEs in this section can all be generalized to apply to both gases and liquids by simply replacing $\gamma$ with $1+B / A$, where $B / A$ is known as the parameter of nonlinearity; see, e.g., Ref. [15], wherein tables containing values of $B / A$ for a wide range of fluids can be found.

Remark 3. Eq. (19) indicates that the value of $\tau$ can (in principle) be computed based on experimental sound speed measurements, i.e., measurements of $\hat{c}_{0}$, in gas-saturated porous media, of the type assumed here, assuming all other material properties are known.

\footnotetext{
4 See, e.g., Ref. [14], and those cited therein, wherein the basis and methodology of this long-established generalization of linear acoustics theory are discussed.
} 


\section{Acceleration wave analysis: the start-up case}

\subsection{Mathematical preliminaries}

We now rescind the small Mach number and related assumptions of the previous section and, henceforth, restrict our attention to planar wave fronts propagating along, and perpendicularly to, the $x$-axis. Under this geometry the velocity vector takes the particular (1D) form $\mathbf{v}=(u(x, t), 0,0)$, the functional form of the condensation becomes $s=s(x, t)$, and the dimensionless versions of Eqs. (5a) and (7) reduce to

$$
\begin{aligned}
& s_{t}+\epsilon\left[u s_{x}+(1+s) u_{x}\right]=0, \\
& (\sigma+s)\left(u_{t}+\epsilon u u_{x}\right)=-\epsilon^{-1}(1+s)^{\gamma-1} s_{x}-\delta u,
\end{aligned}
$$

respectively.

Following the convention adopted by Chen [17, Section 4], we define the amplitude of the jump in a function $\mathfrak{F}=\mathfrak{F}(x, t)$ across a wavefront (i.e., singular surface) $x=\mathfrak{S}(t)$ as

$$
\llbracket \mathfrak{F} \rrbracket:=\mathfrak{F}^{-}-\mathfrak{F}^{+},
$$

where $\mathfrak{F}^{\mp}:=\lim _{x \rightarrow \mathfrak{S}(t)^{\mp}} \mathfrak{F}(x, t)$ are assumed to exist, and where a ' + ' superscript corresponds to the region into which $\mathfrak{S}$ is advancing while a '-' superscript corresponds to the region behind $\mathfrak{S}$.

In this analysis, we assume that $\llbracket u \rrbracket=0$, but that $\llbracket u_{t} \rrbracket \neq 0$. Moreover, we limit our attention to the case of a rightrunning wavefront that is advancing into a motionless gas. As such, $u^{+}=u^{-}=s^{+}=s^{-}=0$ and $u_{t}$ suffers a jump discontinuity across the wavefront

$$
\mathfrak{S}(t)=\hat{U} t+x_{0},
$$

where $\hat{U}(>0)$, the speed of $\mathfrak{S}$ relative to the gas ahead of it, and $x_{0}$, the location of $\mathfrak{S}$ at time $t=0$, are constants, as yet unknown. reads

On taking jumps of the equations in Sys. (21) and introducing Maxwell's theorem [18], which in the present (1D) setting

$$
\llbracket \mathfrak{F}_{t} \rrbracket+\hat{U} \llbracket \mathfrak{F}_{x} \rrbracket=0,
$$

the following jump relations are easily determined:

$$
\llbracket u_{t} \rrbracket=-\hat{U} \llbracket u_{x} \rrbracket, \quad \llbracket s_{t} \rrbracket=-\hat{U} \llbracket s_{x} \rrbracket, \quad \llbracket s_{t} \rrbracket=-\epsilon \llbracket u_{x} \rrbracket, \quad \sigma \llbracket u_{t} \rrbracket=-\epsilon^{-1} \llbracket s_{x} \rrbracket .
$$

In deriving these relations, use was also made of the continuity of $u, s$ and the jump product rule

$$
\llbracket \mathfrak{F} \mathfrak{G} \rrbracket=\mathfrak{G}^{+} \llbracket \mathfrak{F} \rrbracket+\mathfrak{F}^{+} \llbracket \mathfrak{G} \rrbracket+\llbracket \mathfrak{F} \rrbracket \llbracket \mathfrak{G} \rrbracket .
$$

From Eqs. (25) it can be shown that

$$
\hat{U}= \pm \hat{\mathfrak{a}}_{0},
$$

where, in keeping with our assumption of a right-running wavefront, we hereafter neglect the '-' case.

\subsection{Amplitude equation and jump evolution}

Once again taking jumps of the equations in Sys. (21), after now first differentiating them with respect to $t$, and then calling upon Hadamard's lemma $[18,19]$, which in the present context assumes the relatively simple form

$$
\frac{\mathfrak{d} \llbracket \mathfrak{F} \rrbracket}{\mathfrak{d} t}=\llbracket \mathfrak{F}_{t} \rrbracket+\hat{\mathfrak{a}}_{0} \llbracket \mathfrak{F}_{x} \rrbracket,
$$

it can, without great difficulty, be established that the acceleration wave amplitude, $\llbracket u_{t} \rrbracket$, is governed by the following ordinary differential equation (ODE) of the Bernoulli-type:

$$
\frac{\mathfrak{d} \llbracket u_{t} \rrbracket}{\mathfrak{d} t}+\frac{1}{2} \hat{\delta} \llbracket u_{t} \rrbracket-\frac{\epsilon}{2}\left(\frac{\sigma(\gamma+2)-1}{\sqrt{\sigma}}\right) \llbracket u_{t} \rrbracket^{2}=0 .
$$

Here, the 1D displacement derivative $\mathfrak{d} / \mathfrak{d} t$ gives the time-rate-of-change measured by an observer traveling with $\mathfrak{S}$.

As Eq. (29) is exactly reducible to a linear ODE, it is with ease that we obtain the exact solution

$$
\llbracket u_{t} \rrbracket=\frac{\hat{\alpha}^{\star}}{1-\left(1-\frac{\hat{\alpha}^{\star}}{\llbracket u_{t} \rrbracket_{0}}\right) \exp [t(\hat{\delta} / 2)]} .
$$


In Eq. (30),

$$
\hat{\alpha}^{\star}=\frac{\delta}{\epsilon \sqrt{\sigma}[\sigma(\gamma+2)-1]}=\alpha^{\star} g(\sigma),
$$

where we have set

$$
g(\sigma):=\frac{2}{\sqrt{\sigma}\left[2 \sigma+\beta^{-1}(\sigma-1)\right]},
$$

is the critical initial amplitude of the acceleration wave; $\llbracket u_{t} \rrbracket_{0}(\neq 0)$ denotes the value of $\llbracket u_{t} \rrbracket$ at time $t=0$ (i.e., at start-up); and we note that $\alpha^{\star}=\delta /(2 \epsilon \beta)$ is the critical initial amplitude value found in Ref. [3] for the DJM.

Based on Eq. (30), we find that $\llbracket u_{t} \rrbracket$ can evolve over time in any one of the following four (possible) ways:

(I) If $\llbracket u_{t} \rrbracket_{0}<0$, then $\llbracket u_{t} \rrbracket \in\left[-\left|\llbracket u_{t} \rrbracket_{0}\right|, 0\right)$, for all $t \geq 0$, and $\llbracket u_{t} \rrbracket \rightarrow 0$ monotonically from below as $t \rightarrow \infty$.

(II) If $0<\llbracket u_{t} \rrbracket_{0}<\hat{\alpha}^{\star}$, then $\llbracket u_{t} \rrbracket \in\left(0, \llbracket u_{t} \rrbracket_{0}\right]$, for all $t \geq 0$, and $\llbracket u_{t} \rrbracket \rightarrow 0$ monotonically from above as $t \rightarrow \infty$.

(III) If $\llbracket u_{t} \rrbracket_{0}=\hat{\alpha}^{\star}$, then $\llbracket u_{t} \rrbracket=\hat{\alpha}^{\star}$ for all $t \geq 0$.

(IV) If $\llbracket u_{t} \rrbracket_{0}>\hat{\alpha}^{\star}$, then $\lim _{t \rightarrow \hat{t}_{\infty}} \llbracket u_{t} \rrbracket=\infty$, where $\hat{t}_{\infty}(>0)$ is given by

$$
\hat{t}_{\infty}:=2 \hat{\delta}^{-1} \ln \left[\frac{\llbracket u_{t} \rrbracket_{0}}{-\hat{\alpha}^{\star}+\llbracket u_{t} \rrbracket_{0}}\right],
$$

the classical interpretation of which is that a poroacoustic shock wave forms (i.e., $u$ develops a jump discontinuity) at time $t=\hat{t}_{\infty}$.

Here, we observe that an acceleration wave is expansive (resp. compressive) if $\llbracket u_{t} \rrbracket<0$ (resp. $\llbracket u_{t} \rrbracket>0$ ); see Chen [17]. From this, it is not difficult to establish the following:

(a) If $\llbracket u_{t} \rrbracket_{0}<0$, then $\mathfrak{S}$ is expansive for all $t \geq 0$.

(b) If $\llbracket u_{t} \rrbracket_{0} \in\left(0, \hat{\alpha}^{\star}\right]$, then $\mathfrak{S}$ is compressive for all $t \geq 0$.

(c) If $\llbracket u_{t} \rrbracket_{0}>\hat{\alpha}^{\star}$, then $\mathfrak{S}$ is compressive for all $t \in\left[0, \hat{t}_{\infty}\right)$.

Remark 4. If the fluid in question obeys a general barotropic equation of state, say, $\wp=\wp_{0} \Pi(s)$, then $\hat{U}= \pm$ Ma $\times \sqrt{\sigma^{-1}(\partial \Pi / \partial s)^{+}}$and Eq. (29) generalizes to

$$
\frac{\partial \llbracket u_{t} \rrbracket}{\partial t}+\frac{1}{2} \hat{\delta} \llbracket u_{t} \rrbracket-\frac{\epsilon}{2 M a} \sqrt{\frac{\sigma}{(\partial \Pi / \partial s)^{+}}}\left(3-\frac{1}{\sigma}+\frac{\left(\partial^{2} \Pi / \partial s^{2}\right)^{+}}{(\partial \Pi / \partial s)^{+}}\right) \llbracket u_{t} \rrbracket^{2}=0 .
$$

Here, we have introduced the second Mach number Ma $:=c_{0}^{-1} \sqrt{\wp_{0} / \varrho_{0}}$, where we observe that Ma $=\gamma^{-1 / 2}$ in the case of a perfect gas.

Remark 5. For $r \in(0,1)$, where $r:=\alpha^{\star} / \llbracket u_{t} \rrbracket_{0}$, it can be shown that $\hat{t}_{\infty}$ and $t_{\infty}$, where $t_{\infty}=\lim _{\tau \rightarrow 0} \hat{t}_{\infty}$, or from Ref. [3]

$$
t_{\infty}:=2 \delta^{-1} \ln \left[\frac{\llbracket u_{t} \rrbracket_{0}}{-\alpha^{\star}+\llbracket u_{t} \rrbracket_{0}}\right],
$$

are both strictly increasing functions of the ratio $r$; however, $t_{\infty}>\hat{t}_{\infty}$, for every $r \in(0,1)$, since $\sigma>1$.

\subsection{Acceleration wave issues stemming from the formulation of Sys. (1)}

In Ref. [1, Section 4], Ciarletta et al. specialized their analysis to the case of propagation in 1D, under the same geometry adopted in Section 4.1. They discovered, however, that the coefficient ' $b$ ' [1, Eq. (21)] in their jump amplitude equation, the value of which sets the rate of amplitude growth/decay, contains a term proportional to $1 / \tau$, a result which the authors of Ref. [1] found noteworthy. ${ }^{5}$ The presence of this term means that the jump amplitude equation corresponding to the DJM cannot, in general, be recovered from Ref. [1, Eq. (19)] by letting $\tau \rightarrow 0$.

The situation is easier to see when, as in the present section, the gas ahead of the acceleration wave is taken to be at rest. For this special case, $b=1 /(2 \tau)$ [1, Eq. $(22)_{1}$ ], while the corresponding term in Eq. (29) is $\hat{\delta} / 2$. Thus, while the former blows-up as $\tau \rightarrow 0$, the latter tends to $\delta / 2$, which is the correct, corresponding, coefficient under the DJM.

Another implication of the modeling approach adopted in Ref. [1] is that the wave speed corresponding to the DJM cannot be derived from the expression for $U^{2}$ given in Ref. [1, Eq. (15)], where Ciarletta et al. use $U$ to denote the dimensional speed of the acceleration wave; specifically, since $k_{1} \propto 1 / \tau$ is the coefficient of this expression, $U \rightarrow \infty$ in the limit $\tau \rightarrow 0$.

\footnotetext{
5 This behavior stems directly from the fact that Eq. (3), which Ciarletta et al. (in effect) took as their momentum equation, exhibits a drag term (i.e., the last term on the RHS) with coefficient $1 / \tau$.
} 


\section{Traveling wave results}

While a complete traveling wave analysis is not possible here, in this section we present a number of findings, which stem from the traveling wave reduction of Sys. (21), relating to acceleration waves under the JDC model.

\subsection{Associated ODE and phase plane results}

Seeking right-running traveling wave solutions (TWS)s, we now assume

$$
s(x, t)=S(\xi) \text { and } u(x, t)=U(\xi),
$$

where $\xi:=x-c t$ is the wave variable and the wave speed $c$ is a positive constant. On substituting these ansätze into Eqs. (21a) and (21b), and then integrating the former once with respect to $\xi$, Sys. (21) becomes, after simplifying,

$$
\begin{aligned}
& U=\frac{c S}{\epsilon(1+S)}, \\
& \sigma(1+S / \sigma)(c-\epsilon U) U^{\prime}=\epsilon^{-1}(1+S)^{\gamma-1} S^{\prime}+\delta U,
\end{aligned}
$$

where a prime denotes $d / d \xi$. Here, the resulting constant of integration is necessarily zero since, in keeping with Jordan and Fulford's [10] treatment of the (exact) DJM, we have also assumed that the gas at $\xi=-\infty$ is in its equilibrium state. On eliminating $U$ between its two equations, Sys. (37) is readily reduced to the following first order ODE:

$$
\left[\sigma(1+S / \sigma)-c^{-2}(1+S)^{\gamma+2}\right] S^{\prime}=c^{-1} \delta S(1+S)^{2},
$$

which we note is an associated ODE of Sys. (21).

A phase plane analysis reveals both the qualitative behavior of $S$ and the asymptotic nature of the equilibria of Eq. (38). Omitting the trivial case corresponding to $S(\xi)=0$ everywhere on the $\xi$-axis, we observe that Eq. (38) admits three propagation regimes ${ }^{6}$; here, however, we shall consider only the following two:

(i) If $c>\hat{\mathfrak{a}}_{0}$ and $S_{\mathrm{w}} \in(-1,0)$, then the integral curves assume the form of kinks [21], such that $S(\xi) \in(-1,0)$ for every $\xi \in \mathbb{R}$, whose two equilibria, i.e., $\bar{S}=\{-1,0\}$, are asymptotically stable and unstable, respectively.

(ii) If $c=\hat{\mathfrak{a}}_{0}$ and $S_{\mathrm{w}} \in(-1,0)$, then the integral curves assume the form of semi-compact kinks [22], such that $S(\xi) \in$ $(-1,0]$ for every $\xi \in \mathbb{R}$, for which the equilibrium point $\bar{S}=-1$ is asymptotically stable.

Here, $S_{\mathrm{w}}$ denotes the value of $S$ at the wavefront $\xi=0$ (i.e., $S(0)=S_{\mathrm{w}}$ ) and we recall that $\hat{\mathfrak{a}}_{0}=1 / \sqrt{\sigma}$.

\subsection{Case (i): $c>1 / \sqrt{\sigma}$}

In this case Eq. (38) yields, on separating variables and integrating using the software package MATHEMATICA (ver. 9), the exact, but implicit, solution

$$
\begin{aligned}
\xi= & \frac{c}{\delta}\left\{\left[\frac{\sigma-1}{1+\Theta}+\sigma \ln (-\Theta)-\sigma \ln (1+\Theta)+c^{-2}(\gamma+1)^{-1}\right.\right. \\
& \left.\left.\times F(1, \gamma+1 ; \gamma+2 ; 1+\Theta)(1+\Theta)^{\gamma+1}\right]\left.\right|_{\mathcal{S}_{\mathrm{w}}} ^{S}\right\} \quad(-1<S<0),
\end{aligned}
$$

where $F(h, i ; j ; \varsigma)$ denotes the Gauss hypergeometric series [23, Section 15]. Eq. (40), we observe, is the $\tau>0$ generalization of Ref. [10, Eq. (3.12)].

As the integral curves for this case are kinks, a shock thickness can be defined. Adopting the same definition used in Ref. [10], we find, on setting $S=1 / 2$ in Eq. (38) and solving for $S^{\prime}$, that

$$
\hat{l}=\frac{4}{c \delta}\left[c^{2}(2 \sigma-1)-2^{-(\gamma+1)}\right] .
$$

Here, we observe that $\hat{l}>0$ and, moreover, that $\hat{l} \rightarrow l$ as $\tau \rightarrow 0$, where $l$ is the shock thickness corresponding to the exact DJM (see Ref. [10, Eq. (4.7)]). For fixed $c>1>\hat{\mathfrak{a}}_{0}\left(=\sigma^{-1 / 2}\right)$, it is clear that $\hat{l}$ is a (linearly) increasing function of $\sigma(>1)$. Eq. (40) also makes clear that the integral curves generated by Eq. (39) 'shock-up' as $\delta \rightarrow \infty$; on this point, however, see Ref. [10, Section 4.3.1].

\footnotetext{
6 The third case, which corresponds to $c<\hat{\mathfrak{a}}_{0}$, exhibits dual-valued integral curves, the physical interpretation of which is propagating shock waves; see, e.g., Whitham [13, p. 77], as well as Refs. [20, Section 5.2.4] and [10, Section 4.3.2] and those cited therein.
} 
With regard to the velocity field it is easily shown, using Eqs. (37a) and (39), that if $S_{\mathrm{w}} \in(-1,0)$, then

$$
-\infty<U(\xi)<0 \quad(-\infty<\xi<+\infty),
$$

which is also true of the velocity field under the DJM.

On the other hand, the relation

$$
U^{\prime}=\frac{c S^{\prime}}{\epsilon(1+S)^{2}},
$$

which is easily derived from Eq. (37a), can, with the aid of Eq. (38), be recast as

$$
U^{\prime}=\frac{\delta S}{\epsilon\left[\sigma(1+S / \sigma)-c^{-2}(1+S)^{\gamma+2}\right]} .
$$

If we now take, in turn, the limits $S \rightarrow\{0,-1\}$ in Eq. (43), we find that

$$
0<A(\xi)<\frac{c \delta}{\epsilon(\sigma-1)} \quad(c>1 / \sqrt{\sigma}),
$$

where, in the traveling wave context, the acceleration (recall Section 4 ) is given by

$$
A(\xi)=-c U^{\prime}(\xi) .
$$

Eq. (44) indicates that, while it is not under the exact DJM, $A(\xi)$ is a bounded function under the reformulated DJC model.

\subsection{Case (ii): $c=1 / \sqrt{\sigma}$}

While the integral curve in this case is continuous everywhere on the $\xi$-axis, its slope is not. To understand the nature of this discontinuity exhibited by $S^{\prime}$, we take advantage of the fact that $|S|<1$ and re-express the $c=1 / \sqrt{\sigma}$ case of Eq. (38) as

$$
\left[\sigma^{-1}-(\gamma+2)\right] s\left\{\left[1-\left(\frac{(\gamma+2)(\gamma+1)}{2 / \sigma-2(\gamma+2)}\right) s-\cdots\right] s^{\prime}-\frac{\delta \sqrt{\sigma}(1+s)^{2}}{1-\sigma(\gamma+2)}\right\}=0,
$$

from which it is clear that $S=0$ is a solution; however,

$$
\lim _{S \rightarrow 0} \frac{\delta \sqrt{\sigma} S(1+S)^{2}}{(1+S / \sigma)-(1+S)^{\gamma+2}}=\frac{-\delta \sqrt{\sigma}}{\sigma(\gamma+2)-1} \quad(c=1 / \sqrt{\sigma}) .
$$

Integrating this special case of Eq. (38) requires, of course, that the cases $S=0$ and $S \neq 0$ be treated separately. Omitting the details, it is a straightforward matter to obtain the following piecewise-defined solution profile:

$$
\begin{aligned}
S(\xi)= & 0, \quad \xi \in\left(-\infty, \xi_{0}\right], \\
\xi= & \frac{\sqrt{\sigma}}{\delta}\left\{\left[\frac{\sigma-1}{\sigma(1+S)}+\ln (-S)-\ln (1+S)+(\gamma+1)^{-1}\right.\right. \\
& \left.\left.\times F(1, \gamma+1 ; \gamma+2 ; 1+S)(1+S)^{\gamma+1}\right]\left.\right|_{S_{w}} ^{S}\right\}, \quad s \in(-1,0),
\end{aligned}
$$

where we note that for this case $S \in(-1,0)$ implies $\xi>\xi_{0}$. Here, we have set

$$
\begin{aligned}
\xi_{0}:= & \frac{\sqrt{\sigma}}{\delta}\left[\left(\frac{\sigma-1}{\sigma}\right) \frac{S_{\mathrm{w}}}{1+S_{\mathrm{w}}}-\lambda-\psi(\gamma+1)-\ln \left(-S_{\mathrm{w}}\right)+\ln \left(1+S_{\mathrm{w}}\right)\right. \\
& \left.-(\gamma+1)^{-1} F\left(1, \gamma+1 ; \gamma+2 ; 1+S_{\mathrm{w}}\right)\left(1+S_{\mathrm{w}}\right)^{\gamma+1}\right],
\end{aligned}
$$

where $\psi(\varsigma)$ is the digamma function [23, Section 6$]$ and $\lambda \approx 0.5772$ denotes the Euler-Mascheroni constant. We observe that the shock thickness expression for the semi-compact kinks that are the integral curves of Eq. (48) is easily obtained by letting $c \rightarrow 1 / \sqrt{\sigma}$ in Eq. (40).

Now, from Eqs. (47) and (48) it is readily established that

$$
\llbracket S^{\prime} \rrbracket=\left(S^{\prime}\right)^{-}-\left(S^{\prime}\right)^{+}=\frac{\delta \sqrt{\sigma}}{\sigma(\gamma+2)-1},
$$

where in the present context $\mathfrak{S}(t)=c t+\xi_{0}$, i.e., the wavefront of the acceleration wave is located at $\xi=\xi_{0}$. 

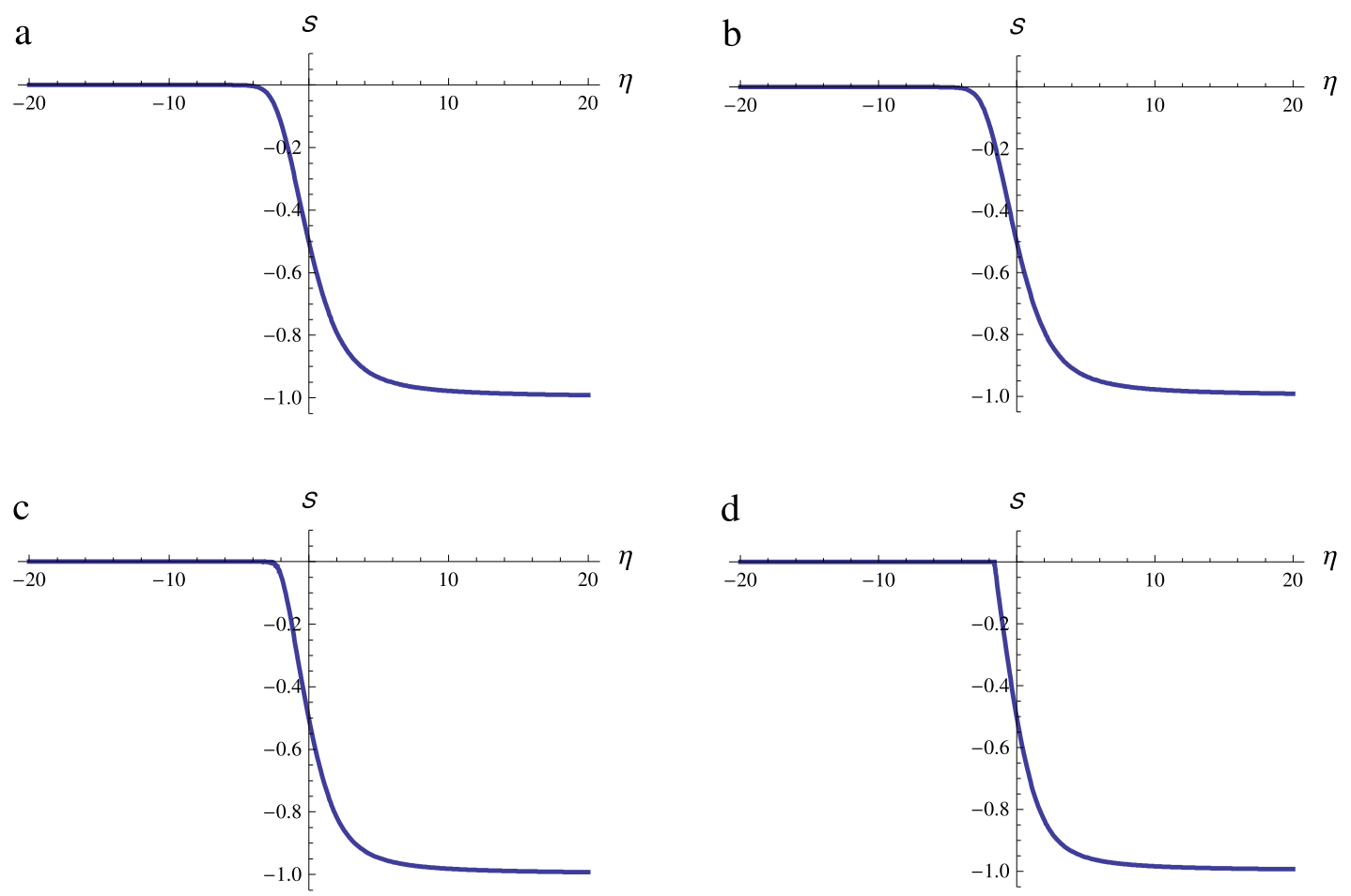

Fig. 1. $S$ vs. $\eta$, where $\eta:=\delta \xi$, for $\epsilon=0.1, \gamma=5 / 3, \sigma=1.1$, and $S_{w}=-0.5$. Here, Fig. 1(a)-(d) correspond to $c / \hat{\mathfrak{a}}_{0}=c \sqrt{\sigma}=1.5,1.25,1.1,1.0001$, respectively.

If we now let $S \rightarrow 0$ in the $c=1 / \sqrt{\sigma}$ special case of Eq. (42), and make use of Eq. (47), then it is not difficult to show that

$$
\llbracket A \rrbracket=\frac{-\delta}{\epsilon \sqrt{\sigma}[\sigma(\gamma+2)-1]}=-\hat{\alpha}^{\star} \quad(c=1 / \sqrt{\sigma}) ;
$$

that is, $-\llbracket A \rrbracket=\llbracket u_{t} \rrbracket$ when $c=\hat{\mathfrak{a}}_{0}$.

In qualitative terms, the findings presented in this subsection and Eq. (44) can be summarized as follows.

$$
\text { For } c=1 / \sqrt{\sigma}: \begin{cases}A(\xi)=0, & \xi \in\left(-\infty, \xi_{0}\right], \\ A(\xi) \in\left(\hat{\alpha}^{\star}, \frac{\delta}{\epsilon(\sigma-1) \sqrt{\sigma}}\right), & \xi \in\left(\xi_{0},+\infty\right) .\end{cases}
$$

For an example of an acceleration wave-bearing acoustic traveling wave profile in the non-porous case, see Ref. [20, Section 5.2.3].

\subsection{Numerical results}

The figures presented in this subsection were generated based on numerical solutions of Eq. (38), which we obtained using the NDSolve routine found in the software package MATHEMATICA (ver. 9). In all eight plots, the gas phase is assumed to be a monatomic gas (e.g., Ar, He, Xe), for which $\gamma=5 / 3$; see, e.g., Refs. [12,13]. In the case of Fig. 2, use was also made of Eqs. (43) and (45).

The sequence shown in Fig. 1 illustrates part of the transition of the $S$ vs. $\eta$ solution profile $(\eta:=\delta \xi)$ from $C^{\infty}(\mathbb{R})$ kink to $C^{0}(\mathbb{R})$ (but not $C^{1}(\mathbb{R})$ ) semi-compact kink as $c \rightarrow 1 / \sqrt{\sigma}$ (from above). Especially noteworthy is the formation of a corner, which in the actual limit is located at $\eta \approx-1.620$, that signifies the fact that the slope of the integral curve corresponding to the $c=1 / \sqrt{\sigma}$ case exhibits a jump discontinuity (i.e., an acceleration wave).

The sequence shown in Fig. 2 captures a subset of the aforementioned transition from the perspective of the $A$ vs. $\xi$ profile. Note that like those of the condensation, the integral curves of the acceleration field assume the general form of kinks, where their asymptotic limits are given by Eqs. (44) and (52) for the cases $c>1 / \sqrt{\sigma}$ and $c=1 / \sqrt{\sigma}$, respectively. Of course, the corner seen forming in Fig. 1 manifests itself as a (forming) jump discontinuity in Fig. 2, where we observe that $\llbracket A \rrbracket=-\hat{\alpha}^{\star} \approx-0.346$ and $\xi_{0} \approx-14.731$ when the limit is actually taken. 

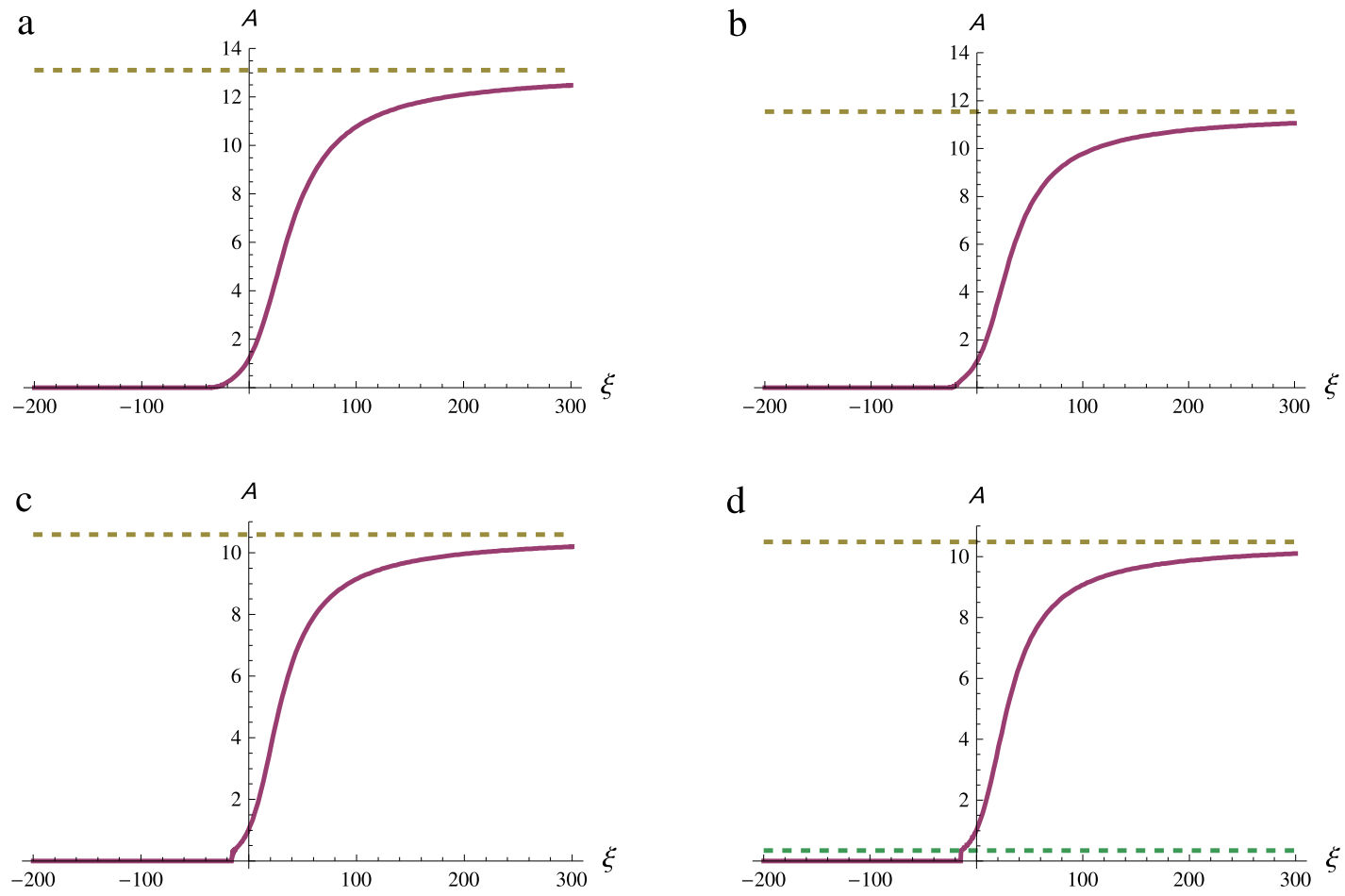

Fig. 2. $A$ vs. $\xi$ for $\epsilon=0.1, \gamma=5 / 3, \sigma=1.1, \delta=0.11$, and $S_{\mathrm{w}}=-0.5$. Here, Fig. 2(a)-(d) correspond to $c / \hat{\mathfrak{a}}_{0}=c \sqrt{\sigma}=1.25,1.1,1.01,1.0001$, respectively; the gold and green dashed lines correspond to $A=c \delta /(\epsilon \sigma-\epsilon)$ and $A=\hat{\alpha}^{\star}$, respectively. (For interpretation of the references to color in this figure legend, the reader is referred to the web version of this article.)

Remark 6. While (the equivalent of) $U_{w}>0$ was assumed in Ref. [24] for simplicity of presentation, the more recent work of Jordan and Fulford [10], who obtained (1D) TWSs for the $\sigma:=1$ (i.e., $\tau:=0$ ) special case of Sys. (21), has shown that it is actually $U_{\mathrm{w}}<0$, which corresponds to $S_{\mathrm{w}} \in(-1,0)$ here, that yields a flow scenario that is physically tenable; specifically, poroacoustic traveling waves, propagating to the right along the $x$-axis, where at the left-asymptotic limit the gas is in its equilibrium state while at the right-asymptotic limit there exists the vacuum state, ${ }^{7}$ and thus a breakdown of the continuum assumption, a consequence of which is the blow-up of $U$ noted in Eq. (41). (For an example of vacuum state-induced velocity blow-up in the context of linear acoustics, see Ref. [13, p. 160].)

\section{Closure}

We have put forth and, in the context of both acceleration waves and traveling waves, analyzed an alternative formulation of the JDC model. In contrast to Ciarletta et al. [1], who treated Eq. (1b) as a momentum balance equation, we regard Eq. (1b) as a constitutive relation and, in accordance with mixture theory [9], take its LHS as the interaction term in the usual Euler momentum equation; see Eq. (5b). Sys. (5), our reformulation of the JDC model, not only generalizes the DJM $[3,10]$ to the case $\tau>0$, but it also allowed us to resolve suspect findings yielded by the acceleration wave analysis carried out in Ref. [1]; see Section 4.3.

Additionally, we have generalized the EoM that is the weakly-nonlinear DJM to include the effects of $\tau$ (see Section 3 ) and, moreover, we have shown that, while $\hat{l}>l$, the quantities $\hat{\mathfrak{a}}_{0}, \hat{\alpha}^{\star}, \hat{\delta}$, and $\hat{t}_{\infty}$, are all less than their counterparts in the exact DJM; in particular, $\hat{\mathfrak{a}}_{0} \in(0,1)$.

More significantly, however, our analysis has revealed that under the reformulated JDC model the $A$ vs. $\xi$ profile is a kink (see Fig. 2), and thus bounded; again, see Eqs. (44) and (52). That is, generalizing the DJM to include the case $\tau>0$ regularizes the otherwise unbounded (from above) acceleration field in the traveling wave context. This regularization vis-à-vis the DJM does not, unfortunately, extend to $U$, the velocity field. To achieve this, one must add the 'Brinkman term' [4,19], specifically, $-\mu_{\text {eff }} \chi \nabla^{2} \mathbf{v}$, where $\mu_{\text {eff }}(>0)$ is an effective viscosity coefficient, to the LHS of Eq. (5b). While this modification yields $U$ vs. $\xi$ profiles in the form of kinks, as Rossmanith and Puri [25] have recently demonstrated in the case of the weakly-nonlinear DJM, it also destroys the model's hyperbolicity, and thus its ability to satisfy the requirements of causality.

7 That is, $S(\xi) \rightarrow-1$ as $\xi \rightarrow+\infty$, meaning that the mass density of the gas tends to zero as $\xi \rightarrow+\infty$, an aspect of Sys. (21) that the weakly-nonlinear EoMs in Section 3 cannot be expected to fully capture; recall the assumption $|s| \ll 1$. 
And finally, along with including the Brinkman term, and those suggested in Ref. [10, Section 5], we offer the following as a possible generalization, which might prove to be tractable (in 1D, at least) from the analytical standpoint, of the reformulated JDC model: In place of Eq. (1b), take the filtration law to be

$$
\left(1+\lambda_{v} \frac{\mathfrak{D}}{\mathfrak{D} t}\right) \mathbf{v}=-\frac{K}{\mu \chi}\left(1+\lambda_{P} \frac{\mathfrak{D}}{\mathfrak{D} t}\right) \nabla P,
$$

which is, of course, a generalization ${ }^{8}$ of Eq. (4). Note that in the case of Eq. (53), our (mixture theoretic-based) momentum equation becomes

$$
\left(1+\lambda_{P} \frac{\mathfrak{D}}{\mathfrak{D} t}\right)\left(\varrho \frac{D \mathbf{v}}{D t}+\nabla \wp\right)=-\frac{\mu \chi}{K}\left\{\lambda_{v}\left[\frac{D \mathbf{v}}{D t}-(\mathbf{v} \cdot \nabla) \mathbf{v}+(\nabla \cdot \mathbf{v}) \mathbf{v}\right]+\mathbf{v}\right\},
$$

which we observe is of second order in time.

\section{Acknowledgments}

The authors thank Profs. Andrew Norris and Michail Todorov for their kind invitation to contribute to this special issue. The authors also thank the two anonymous referees; their valuable comments and suggestions have led to a marked improvement in this article. P.M.J. was supported by ONR funding.

\section{References}

[1] M. Ciarletta, B. Straughan, V. Zampoli, Poroacoustic acceleration waves in a Jordan-Darcy-Cattaneo material, Internat. J. Non-Linear Mech. 52 (2013) $8-11$.

[2] M. Ciarletta, B. Straughan, Poroacoustic acceleration waves, Proc. R. Soc. A 462 (2006) 3493-3499.

[3] P.M. Jordan, Growth and decay of acoustic acceleration waves in Darcy-type porous media, Proc. R. Soc. A 461 (2005) $2749-2766$.

[4] D.A. Nield, A. Bejan, Convection in Porous Media, second ed., Springer, 1999 (Chapter 1).

[5] K.R. Rajagopal, On a hierarchy of approximate models for flows of incompressible fluids through porous solids, Math. Models Methods Appl. Sci. 17 (2007) 215-252.

[6] C.I. Christov, On frame indifferent formulation of the Maxwell-Cattaneo model of finite-speed heat conduction, Mech. Res. Comm. 36 (2009) 481-486.

[7] M. Ciarletta, B. Straughan, V. Tibullo, Christov-Morro theory for non-isothermal diffusion, Nonlinear Anal. RWA 13 (2012) 1224-1228.

[8] A. Morro, Evolution equations and thermodynamic restrictions for dissipative solids, Math. Comput. Modelling 52 (2010) 1869-1876.

[9] K.R. Rajagopal, L. Tao, Mechanics of Mixtures, in: Series on Advances in Mathematics for Applied Sciences, vol. 35, World Scientific, 1995 (Appendix B)

[10] P.M. Jordan, J.K. Fulford, A note on poroacoustic traveling waves under Darcys law: Exact solutions, Appl. Math.-Czech. 56 (2011) 99-115.

[11] B. Khuzhayorov, J.-L. Auriault, P. Royer, Derivation of macroscopic filtration law for transient linear viscoelastic fuid flow in porous media, Internat. J. Engrg. Sci. 38 (2000) 487-504.

[12] P.A. Thompson, Compressible-Fluid Dynamics, McGraw-Hill, 1972

[13] G.B. Whitham, Linear and Nonlinear Waves, Wiley, 1974.

[14] P.M. Jordan, A survey of weakly-nonlinear acoustic models: 1910-2009, Mech. Res. Comm. 73 (2016) 127-139.

[15] R.T. Beyer, The parameter B/A, in: M.F. Hamilton, D.T. Blackstock (Eds.), Nonlinear Acoustics, Academic Press, 1997, pp. 25-39.

[16] F. Coulouvrat, On the equations of nonlinear acoustics, J. Acoustique 5 (1992) 321-359.

[17] P.J. Chen, Growth and decay of waves in solids, in: S. Flügge, C. Truesdell (Eds.), Handbuch der Physik, Vol. VIa/3, Springer, 1973, pp. $303-402$.

[18] C. Truesdell, R.A. Toupin, The classical filed theories, in: S. Flügge (Ed.), Handbuch der Physik, Vol. III/1, Springer, 1960, pp. 491-529.

[19] B. Straughan, Stability and Wave Motion in Porous Media, in: Applied Mathematical Sciences, vol. 165, Springer, 2008.

[20] I.C. Christov, P.M. Jordan, S.A. Chin-Bing, A. Warn-Varnas, Acoustic traveling waves in thermoviscous perfect gases: Kinks, acceleration waves, and shocks under the Taylor-Lighthill balance, Math. Comput. Simul. 127 (2016) 2-18.

[21] J. Angulo, Nonlinear Dispersive Equations: Existence and Stability of Solitary and Periodic Travelling Wave Solutions, in: Mathematical Surveys and Monographs, vol. 156, American Mathematical Society, 2009.

[22] M. Destrade, G. Gaeta, G. Saccomandi, Weierstrass’s criterion and compact solitary waves, Phys. Rev. E 75 (2007) 047601.

[23] M. Abramowitz, I.A. Stegun (Eds.), Handbook of Mathematical Functions, Dover, 1965.

[24] P.M. Jordan, Finite-amplitude acoustic traveling waves in a fluid that saturates a porous medium: Acceleration wave formation, Phys. Lett. A 355 (2006) 216-221.

[25] D. Rossmanith, A. Puri, Recasting a Brinkman-based acoustic model as the damped Burgers equation, Evol. Equ. Control Theory (EECT) 5 (2016) $463-474$.

8 Achieved by replacing $\partial / \partial t$ with $\mathfrak{D} / \mathfrak{D} t$, so that Eq. (53) reduces to Eq. (1b) when $\lambda_{P}=0$. 